\title{
Sensorimotor Gating and Dopamine Function in Postpartum Rats
}

\author{
Elizabeth M Byrnes*,', Robert S Bridges', Victoria F Scanlan', Jessica A Babb' and John J Byrnes² \\ 'Department of Biomedical Sciences, Tufts University Cummings School of Veterinary Medicine, North Grafton, MA, USA; \\ ${ }^{2}$ Department of Neuroscience, Tufts University School of Medicine, Center for Neuroscience Research, Boston, MA, USA
}

\begin{abstract}
There is much speculation regarding the effects of estrogen withdrawal at the end of pregnancy on forebrain dopamine, however, few studies have directly examine changes in this system postpartum. The present work sought to determine what changes in forebrain dopamine function occur in the postpartum rat. Specifically, prepulse inhibition of the acoustic startle response (PPI) was measured in primiparous female rats on postpartum day 2 (PPD2) or 14 (PPDI4) following treatment with saline or the dopamine D2 agonist, quinpirole. Diestrus (DI) females served as controls. Dopamine content and turnover as well as cyclic AMP (cAMP) accumulation were determined within the nucleus accumbens and dorsal striatum in these same females. In addition, circulating levels of plasma corticosterone, estradiol and progesterone were measured. PPI was significantly disrupted in both postpartum groups. This effect was associated with decreased CAMP content within the nucleus accumbens. Quinpirole treatment $(0.1$ and $0.5 \mathrm{mg} / \mathrm{kg})$ dose-dependently disrupted PPI in DI controls while PPD2 and PPDI4 animals demonstrated reduced sensitivity to the D2 agonist. PPDI4 animals demonstrated increased startle amplitude, an effect that was attenuated by quinpirole treatment. PPDI 4 females were also less sensitive to quinpirole-mediated reductions in DA turnover within the nucleus accumbens and both PPD2 and PPDI 4 females had an attenuated response to the stimulatory effects of quinpirole on corticosterone secretion. Collectively these findings suggest that the postpartum period is associated with reduced sensorimotor gating and altered forebrain DA systems, which may be related to shifts in circulating hormones.
\end{abstract}

Neuropsychopharmacology (2007) 32, I021-1031. doi:10.1038/sj.npp. I301222; published online I8 October 2006

Keywords: corticosterone; D2 receptor; estrogen; lactation; prepulse inhibition; progesterone

\section{INTRODUCTION}

Postpartum psychosis is characterized by confusion, disorientation, psychomotor disturbances, depression, mania, hallucinations and delusions (Kohl, 2004). As this condition is responsive to treatment with antipsychotic medications, dopaminergic dysfunction has been proposed as an underlying process in the manifestation of this disorder. The condition typically occurs within 4-6 weeks of birth with an incidence of $0.1-0.5 \%$ (Seyfried and Marcus, 2003). As a precipitous decline in estrogen occurs in the early postpartum period, estrogen withdrawal has been proposed as a mechanism for the onset of psychosis (Huber et al, 2001; Meakin et al, 1995). In the clinical setting, several attempts have been made to decrease the risk of relapse via the administration of postpartum

*Correspondence: Dr EM Byrnes, Department of Biomedical Sciences, Tufts University Cummings School of Veterinary Medicine, 200 Westboro Road, North Grafton, MA 01536, USA, Tel: + I 508839 7986, Fax: + I 508839 709I, E-mail: elizabeth.byrnes@tufts.edu Received 26 January 2006; revised 22 August 2006; accepted 24 August 2006

Online publication: 12 September 2006 at http://www.acnp.org/ citations/Npp09 | 206060049/default.pdf estrogen to women with a history of postpartum psychosis (Ahokas and Aito, 1999; Ahokas et al, 2000). However, these studies have involved relatively small sample sizes, and the results have been equivocal. Nevertheless, the estrogen withdrawal hypothesis of puerperal psychosis remains one of the most dominant premises in the psychiatric literature.

Alterations in the hormonal environment are critical for pregnancy and parturition, and serve to ready the body and brain for the tasks associated with motherhood (eg, lactation and maternal behavior). During pregnancy, levels of estrogen increase as gestation advances. Postpartum, however, the high levels of estrogen decline precipitously. These hormonal fluctuations occur in the vast majority of women in the absence of negative psychiatric outcomes. Thus, it is unclear why these hormonal changes may trigger psychosis in a subset of women. There is little of data regarding the neural adaptations that normally occur in response to pregnancy and parturition. Perhaps by better understanding the characteristic changes associated with the postpartum period, and especially those related to neural dopamine systems, insights may be gained into the potential failures of these processes during the postpartum period in vulnerable populations. 
Prepulse inhibition of the acoustic startle response (PPI) is a functional measure of sensorimotor gating that is observed across species (Swerdlow et al, 2000). Briefly, PPI is the normal attenuation of the startle reflex, which occurs when a stimulus that normally elicits a robust startle response is preceded by a weaker stimulus. PPI is deficient in a number of neuropsychiatric conditions (eg, schizophrenia, obsessive-compulsive disorder, Tourettes syndrome) that are characterized by defective cognitive, motor or sensorimotor inhibition (for review see Braff et al, 2001). Currently no studies have examined PPI in women with postpartum psychosis. However, studies have shown that variations in circulating hormones can modulate PPI (Swerdlow et al, 1997; Koch, 1998) suggesting an interaction between the neural systems that regulate sensorimotor gating and endocrine status.

The administration of dopamine receptor agonists produces deficits in PPI in both humans and rodents. Furthermore, activation of dopamine D2-receptors within the nucleus accumbens is critical for dopamine-mediated disruptions in PPI (Wan et al, 1994; Wan and Swerdlow, 1993; Swerdlow et al, 1990). A previous study examining the long-term effects of pregnancy on forebrain dopamine function found that primiparous females (ie, females that had given birth and reared one litter) were more sensitive to the effects of the dopamine agonist apomorphine on PPI (Byrnes et al, 2001). As that study was performed several weeks after weaning, it remains unknown when this shift in dopamine sensitivity may occur. By measuring PPI in postpartum females in the absence and presence of a dopamine D2-receptor agonist, however, one can observe both the normal alterations in sensorimotor gating that may emerge postpartum as well as potential shifts in dopamine D2-receptor sensitivity within the forebrain dopamine regions that modulate PPI.

The aim of the present study is to assess postpartum changes in sensorimotor gating by measuring PPI in female rats during the early and mid-postpartum period (postpartum days 2 and 14) as compared to non-lactating, diestrus controls. In addition, we also examined the ability of the dopamine D2-agonist, quinpirole, to disrupt PPI as a functional measure of forebrain dopamine sensitivity in postpartum and diestrus females. Quinpirole-induced alterations in locomotor activity were also assessed as an additional behavioral measure of forebrain dopamine sensitivity. Following behavioral testing, dopamine utilization in the nucleus accumbens and dorsal striatum was determined as a presynaptic measure of dopaminergic function. In addition, cAMP accumulation in the nucleus accumbens and dorsal striatum was determined as a postsynaptic measure of dopaminergic activity, as D2receptor activation is known to decrease cAMP accumulation. The current study also measured plasma corticosterone immediately after the completion of behavioral testing to determine whether any of the effects on PPI observed in the postpartum females were due to alterations in corticosterone secretion in response to the testing conditions as lactating females have been found to be stress hyporesponsive under some conditions (Lightman and Young, 1989). Moreover, as D2-agonists have been found to stimulate corticosterone secretion (Borowsky and Kuhn, 1992; Foreman et al, 1989; Fuller et al, 1983) via their effects within the ventral striatum (Ikemoto and Goeders, 1998), quinpirole-stimulated corticosterone secretion served as yet another measure of D2 receptor sensitivity within the forebrain dopamine system. Finally, plasma estradiol and progesterone levels were determined in all subjects in an attempt to correlate behavioral and neurochemical changes with postpartum shifts in hormone levels.

\section{DESIGN AND METHODOLOGY}

\section{Animals and Mating}

Seventy-two virgin female Sprague-Dawley rats (Crl:CD[SD]BR; 176-200 g) were purchased from Charles River Laboratories (Kingston, NY). Following arrival, all animals were allowed to acclimate to the colony for 1 week before mating. Animals were housed in polypropylene cages $(45 \times 25 \times 20 \mathrm{~cm})$ with food and water available ad libitum in light (on 0500-1900 h) - and temperature $\left(21-25^{\circ} \mathrm{C}\right)$ - controlled rooms. Animals were mated in our colony and pregnant females were then housed individually for the duration of the experiment. On postpartum day 1 (birth $=$ day 0 ), all litters were culled to 10 pups. Agematched nulliparous control rats were group-housed throughout the experiment. All procedures were reviewed and approved by the Tufts University Cummings School of Veterinary Medicine Institutional Animal Care and Use Committee, and conducted in accordance the National Research Council Guide for the Care and Use of Laboratory Animals.

\section{Experimental Design and Procedure}

Postpartum lactating females were studied at either postpartum day 2 (PPD2) or postpartum day 14 (PPD14). These time points represent early and mid-postpartum, respectively. Age-matched nulliparous females served as controls and all of these controls were studied on diestrus (DI) as previous studies have observed variations in PPI across the estrous cycle (Koch, 1998). All behavioral testing and tissue collection was conducted between 0800 and $1500 \mathrm{~h}$ with time of day counterbalanced across both treatment groups and drug treatment. On the day of testing, animals were transported to a quiet behavioral testing room and allowed to acclimate to the environment for $5 \mathrm{~min}$. Before the beginning of testing all subjects were weighed. Each animal was then tested for $15 \mathrm{~min}$ in the open field. Animals in each group were then treated with either quinpirole $(0.1$ or $0.5 \mathrm{mg} / \mathrm{kg}$, s.c.; Sigma Chemical Co., Natick, MA) or vehicle (saline, s.c.) and placed back into the open field for an additional $15 \mathrm{~min}$ test. As it was not know whether postpartum females would demonstrate increased or decreased sensitivity to the dopamine agonist, the doses were chosen to include a low dose that does not normally disrupt PPI (to test for increased sensitivity) as well as an intermediate dose that normally disrupts PPI (to test for decreased sensitivity) (Swerdlow et al, 2000). Immediately following activity testing ( $15 \mathrm{~min}$ postdrug treatment), animals were placed into an acoustic startle chamber for PPI testing. The total time of PPI testing was $27 \mathrm{~min}$ after which animals were immediately transported to a necropsy room where they were euthanized and tissue was collected. 


\section{Behavioral Testing}

Open field activity. Animals were removed from the home cage and placed individually into an open field arena (Plexiglas cage; $41 \mathrm{~L} \times 41 \mathrm{~W} \times 38 \mathrm{H} \mathrm{cm}$ ) for $15 \mathrm{~min}$. Motor activity was monitored with the SmartFrame ${ }^{\circledR}$ Open Field Activity System (Hamilton-Kinder, Poway, CA). The frame, which consisted of 32 photocells $(16 \mathrm{~L} \times 16 \mathrm{~W}$, spaced $2.5 \mathrm{~cm}$ apart), surrounded the Plexiglas cage and continuously tracked the animal's movement. Data were collected in the form of photobeam breaks using MotorMonitor ${ }^{\mathbb{R}}$ software (Hamilton-Kinder). The peripheral region was defined as $7.5 \mathrm{~cm}$ from any wall, while the center was defined as the $25 \times 25 \mathrm{~cm}$ region in the middle of the field. The percent of time subjects spent in the center of the open field was used as a functional measure of anxiety-like behavior.

Acoustic startle and prepulse inhibition. Animals were tested for PPI using the Startle Monitor System (HamiltonKinder), which consisted of a PC-interfaced sound-attenuated startle chamber, computer interface chassis, and Windows NT-based software for data analysis. The startle chamber (inner dimensions: $27 \mathrm{~W} \times 27 \mathrm{H} \times 16 \mathrm{D} \mathrm{cm}$ ) consisted of a speaker (diameter: $11 \mathrm{~cm}$ ) mounted on the top and a piezoelectric sensing platform on the floor. During testing, animals were placed in an adjustable holder positioned atop the sensing platform, providing only limited restraint while prohibiting ambulation. Animals were allowed a $5 \mathrm{~min}$ acclimation period before the onset of acoustic stimuli. A continuous $70 \mathrm{dBA}$ broad band ('white') background noise was present during the acclimation period and throughout the experiment. Two trial types were used in the experiment: basic startle stimulus of $40 \mathrm{~ms}$, $120 \mathrm{dBA}$ broad band noise pulse, and a prepulse stimulus that consisted of a $40 \mathrm{~ms}, 85 \mathrm{dBA}$ prepulse that preceded the basic startle stimulus by $100 \mathrm{~ms}$. These two stimulus trial types are referred to herein as Pulse and Prepulse trials, respectively. Each animal received 65 stimuli presented in pseudorandom order with a $15 \mathrm{~s}$ interstimulus interval. The first 10 trials consisted of $120 \mathrm{dBA}$ pulse stimuli to habituate animals to the acoustic stimulus. Responses to these habituation trials were not included in the final PPI analysis. Whole body startle responses were recorded in Newtons. The percent of PPI expressed within each test session was calculated using the standard PPI equation which was as follows: (100-(mean Prepulse response/mean Pulse response) $\times 100$ ).

\section{Neurochemical and Endocrine Analyses}

Immediately following behavioral testing, animals were euthanized via $\mathrm{CO}_{2}$ vapor and brains were rapidly removed. Trunk blood was collected in heparinized tubes for subsequent plasma preparation and hormone determination. For HPLC and cAMP determinations, nucleus accumbens (core and shell regions) and dorsal striatum were dissected bilaterally on ice according to the atlas of Paxinos and Watson (1998). Each unilateral brain region was frozen on dry ice and stored at $-80^{\circ} \mathrm{C}$ until analysis. Protein content in tissue was subsequently determined using the Bradford method (Bio-Rad, Hercules, CA).
Measurement of dopamine and dihydroxyphenylacetic acid in tissue homogenates. Frozen tissue from one hemisphere was homogenized in HPLC mobile phase (MDTM70-1332; ESA, Chelmsford, MA) consisting of the following: $\mathrm{NaH}_{2} \mathrm{PO}_{4} 75 \mathrm{mM}$; EDTA $25 \mu \mathrm{M}$; octanesulfonic acid sodium salt $1.7 \mathrm{mM}$; triethylamine $100 \mu \mathrm{l} / \mathrm{l} ; 10 \%$ acetonitrile; $\mathrm{pH} 3.0$ with phosphoric acid. Homogenates were centrifuged at 12000 r.p.m. for $20 \mathrm{~min}$ after which supernatants were removed and subsequently analyzed for dopamine and DOPAC using HPLC-ED. Dopamine and DOPAC content were analyzed using a DECADE Digital Electrochemical Amperometric Detector (Antec Leyden, Netherlands) and the LC1150 Solvent Delivery System (GBC Scientific Equipment, Victoria, Australia) which pumped mobile phase at a rate of $0.2 \mathrm{ml} / \mathrm{min}$. Catecholamines were separated on a ThermoHypersil-Keystone column $(150 \times 2.1 \mathrm{~mm}$, pore size $5.0 \mu \mathrm{m})$. WinChrom Chromatography data management software (GBC Separations, Hubbardston, MA) was used for data analysis. Dopamine and DOPAC ( $\mathrm{ng} / \mathrm{mg}$ protein) were determined based on comparison to an external standard curve, which was generated each day that tissues were processed. Turnover of dopamine for each brain region was calculated using the DOPAC/DA ratio.

Cyclic AMP measurement in tissue homogenates. Frozen brain tissue from one hemisphere was homogenized in 25 volumes of ice-cold $50 \mathrm{mM}$ Tris buffer $(\mathrm{pH}$ 7.4) containing $1 \mathrm{mM}$ EGTA, $3 \mathrm{mM} \mathrm{MgSO}$, and $10 \mathrm{mM}$ glucose. Homogenates were centrifuged at 12000 r.p.m. for $10 \mathrm{~min}$ at $4^{\circ} \mathrm{C}$. The pellets were resuspended and washed twice in buffer. The final pellet will be resuspended in 10 volumes of cold buffer. A competition binding assay was used to determine relative cAMP production (Munirathiam and Yoburn, 1994; Kaplan et al, 1998). For this assay, $50 \mu 150 \mathrm{mM}$ Tris buffer ( $\mathrm{pH}$ 7.4) containing $4 \mathrm{mM}$ EDTA and $4 \mathrm{nM}\left(20000\right.$ c.p.m.) $\left[{ }^{3} \mathrm{H}\right]$ cAMP (Amersham, Arlington Heights, IL) was added to tubes containing $50 \mu \mathrm{l}$ sample supernatant (above). The mixture was vortexed and incubated on ice for $5 \mathrm{~min}$. Following the addition of $100 \mu \mathrm{l}$ Tris/EDTA buffer containing $5 \mu \mathrm{g}$ bovine heart binding protein (Sigma, St Louis, MO), tubes were vortexed and incubated for an additional $2 \mathrm{~h}$ at $4^{\circ} \mathrm{C}$. Tris/ EDTA buffer $(100 \mu \mathrm{l})$ containing $3.5 \%$ activated charcoal and $0.1 \%$ BSA was then added to the incubation. Tubes were then vortexed and centrifuged at 12000 r.p.m. for $6 \mathrm{~min}$ to separate bound from free $\left[{ }^{3} \mathrm{H}\right] \mathrm{cAMP}$. Supernatants were counted by scintillation spectrophotometry. In this assay, lower concentrations of $\left[{ }^{3} \mathrm{H}\right]$ cAMP in the supernatant reflect higher concentrations of endogenous cAMP in tissue. Thus, data were analyzed and presented as relative cAMP concentrations using the following equation: $1 /$ (c.p.m./mg protein).

Corticosterone, estradiol and progesterone radioimmunoassay. Plasma concentrations of corticosterone $(\mathrm{ng} / \mathrm{ml})$, estradiol $(\mathrm{pg} / \mathrm{ml})$, and progesterone $(\mathrm{ng} / \mathrm{ml})$ were determined using commercially available radioimmunoassay kits (Diagnostics Products Corporation, Los Angeles, CA) according to their respective insert protocols.

\section{Data Analyses}

All data were analyzed with one-, two-, or three-way analysis of variance (ANOVA). All post hoc analyses were 
conducted using Tukey's test. Pearson correlations were used to examine Startle Amplitude and Body Weight. Statistical significance was defined as $P<0.05$.

\section{RESULTS}

\section{Open Field Locomotor Activity}

Before drug or vehicle treatment, a 15 min test of open field behavior was conducted. Data were broken into $5 \mathrm{~min}$ blocks and the analysis was conducted using a repeatedmeasures two-way ANOVA. These data are shown in Figure 1 (top panel). On the measure of beam breaks, the ANOVA indicated a main effect of time $(F(2,215)=151.0$, $P<0.001)$ and a trend toward a main effect of reproductive status $(\mathrm{F}(2,215)=2.73, P=0.07)$. There was no significant group $\times$ time interaction. Thus, all groups demonstrated the expected habituation to the open field with no significant difference observed as a function of reproductive status. The trend toward a reproductive status effect may have been due to slightly more rapid habituation to the open field observed in the lactating females. As the tendency to explore the center of the open field is often used as a measure of anxiety-like behavior, in addition to examining overall activity levels an analysis was conducted on the percent of time subjects spent in the center of the open field. The analysis revealed a significant main effect of time $(\mathrm{F}(2,215)=4.94, P<0.01)$ with exploration of the center of the open field decreasing over time, as shown in Figure 1

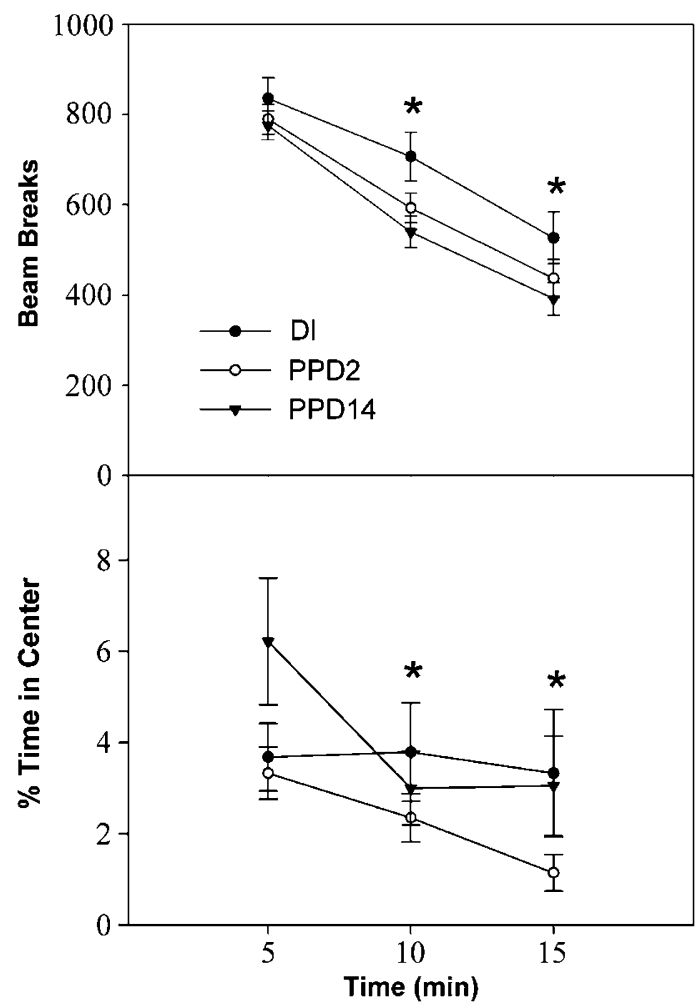

Figure I Mean ( \pm SEM) number of beam breaks (upper panel) and percent of time spent in the center of the open field (lower panel) at $5 \mathrm{~min}$ intervals. $N=24$ /group. $* P<0.05$ as compared to the first $5 \mathrm{~min}$ time period, collapsed across reproductive status group. (bottom panel). There was no effect of reproductive status $(P=0.21)$ and no reproductive status $\times$ time interaction $(P=0.2)$. Thus all three groups appeared to spend an equal amount of time exploring the center of the open field.

A three-way repeated measures ANOVA with reproductive status and quinpirole dose as between subject factors and time as the within-subject factor was conducted on locomotor activity in the open field following quinpirole administration. The analysis revealed a significant main effect of time $(\mathrm{F}(2,130)=42.8, P<0.001)$ as well as a significant time $\times$ dose interaction $(\mathrm{F}(4,130)=6.03$, $P<0.001)$ with no time $\times$ reproductive status effect $(P=0.95)$ and no time $\times$ reproductive status $\times$ dose effect $(P=0.97)$. Tests of between-subjects effects revealed a significant main effect of dose $(\mathrm{F}(2,65)=4.09, P<0.03)$ and a trend toward a main effect of reproductive status $(P=0.08)$ with no reproductive status $\times$ dose effect $(P=0.18)$. The effect of quinpirole on locomotor activity is demonstrated in Figure 2 with the data collapsed across reproductive status. The highest activity levels were observed during the first $5 \mathrm{~min}$ regardless of drug treatment group and are likely a measure of the subject's response to the injection. By the 10 -min period there was a significant reduction in activity in quinpirole-treated animals, which reached significance in animals treated with the $0.1 \mathrm{mg} / \mathrm{kg}$ dose of quinpirole (as compared to saline-treated controls). By the $15 \mathrm{~min}$ period, however, a significant increase in activity was observed in animals treated with the $0.5 \mathrm{mg} / \mathrm{kg}$ dose of quinpirole as compared to both saline- and lowdose-treated animals. These data indicate that locomotor activity in response to quinpirole is both dose and time dependent, but is not influenced by the reproductive status of the female.

\section{Body Weight}

Mean body weight for each group is depicted in Table 1. A two-way ANOVA (reproductive status and quinpirole dose as factors) revealed a significant main effect of reproductive status on body weight $(\mathrm{F}(2,69)=44.27, P<0.01)$ as well as a significant reproductive status $\times$ drug dose interaction

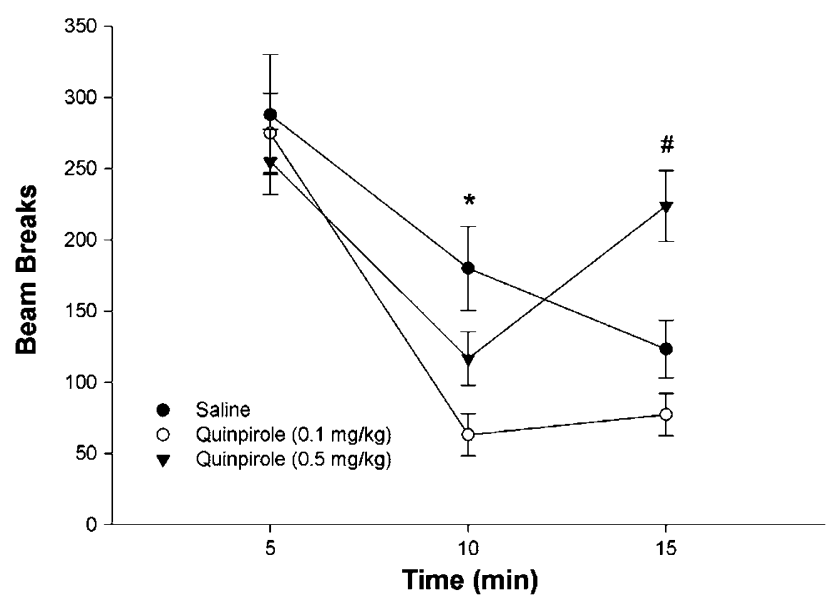

Figure 2 Mean beam breaks $( \pm S E M)$ following quinpirole injection collapsed across reproductive status. $* P<0.05,0.01 \mathrm{mg} / \mathrm{kg}$ vs saline-treated; ${ }^{\#} P<0.05,0.5$ vs saline and $0.1 \mathrm{mg} / \mathrm{kg}$ treated subjects. 
Table I Mean Body Weight in Grams ( \pm SEM) on the Day of Testing

\begin{tabular}{lcc}
\hline Reproductive status & Quinpirole dose (mg/kg) & Body weight (g) \\
\hline $\mathrm{DI}$ & Saline & $278.2 \pm 6.5$ \\
& 0.1 & $264.3 \pm 6.3$ \\
& 0.5 & $283.1 \pm 6.2$ \\
& & \\
PPD2 & Saline & $278.9 \pm 7.7$ \\
& 0.1 & $277.1 \pm 5.3$ \\
& 0.5 & $269.5 \pm 8.3$ \\
PPDI4* & & \\
& Saline & $311.5 \pm 4.4$ \\
& 0.1 & $309.1 \pm 4.6$ \\
& 0.5 & $334.8 \pm 8.2 * *$ \\
\hline
\end{tabular}

$N=7-8$ per group.

* $P<0.0$ l as compared to DI and PPD2 across dose. $* * P<0.05$ as compared to saline and $0.1 \mathrm{mg} / \mathrm{kg}$ within PPDI 4 as well as compared to DI and PPD2 within dose.

$(\mathrm{F}(4,69)=2.55, \quad P<0.05)$. The main effect was due to increased body weight in the PPD14 females compared to those in DI and PPD2 groups $\left(P^{\prime} s<0.01\right)$. Previous reports have also observed significant body weight gain as lactation progresses (Woodside et al, 1998). The interaction, however, was due to higher body weights observed in the PPD14 subjects treated with the higher dose of quinpirole $(P<0.025)$. As subjects were randomly assigned to treatment groups, this effect was unexpected, but requires consideration in the analysis of the effects of reproductive status and drug treatment on startle amplitude.

\section{Acoustic Startle Amplitude}

A two-way ANOVA with reproductive status and quinpirole dose as factors was conducted using the mean startle amplitude during the pulse alone trials. These data revealed a significant main effect of reproductive experience on startle amplitude $(\mathrm{F}(2,67)=8.14, P<0.001)$ as well as a main effect of quinpirole dose $(\mathrm{F}(2,67)=6.03, P<0.01)$ and a marginally significant interaction $(F(4,67)=3.40$, $P=0.06)$. As illustrated in Figure 3 , startle amplitude was significantly increased in PPD14 females when compared to both PPD2 females and DI controls (both $P^{\prime} s<0.01$ ). The quinpirole dose effect was due to decreased startle amplitude following the higher dose of quinpirole $(0.5 \mathrm{mg} /$ $\mathrm{kg})$ as compared to saline $(P<0.01)$, however, post hoc analyses indicated that the effect of quinpirole on startle amplitude was only significant in PPD14 females. It is unlikely that these differences were the result of the increased body weights as a Pearson correlation performed to determine whether bodyweight and startle amplitude covaried in PPD14 females was not significant $(P>0.05)$. Moreover, the lowest startle amplitudes were observed in the PPD14 females with the highest bodyweights (ie, $0.5 \mathrm{mg} /$ $\mathrm{kg}$ quinpirole-treated).

As the findings on startle amplitude were unexpected, we decided to explore this effect further and examine potential changes in startle amplitude across the testing procedure in

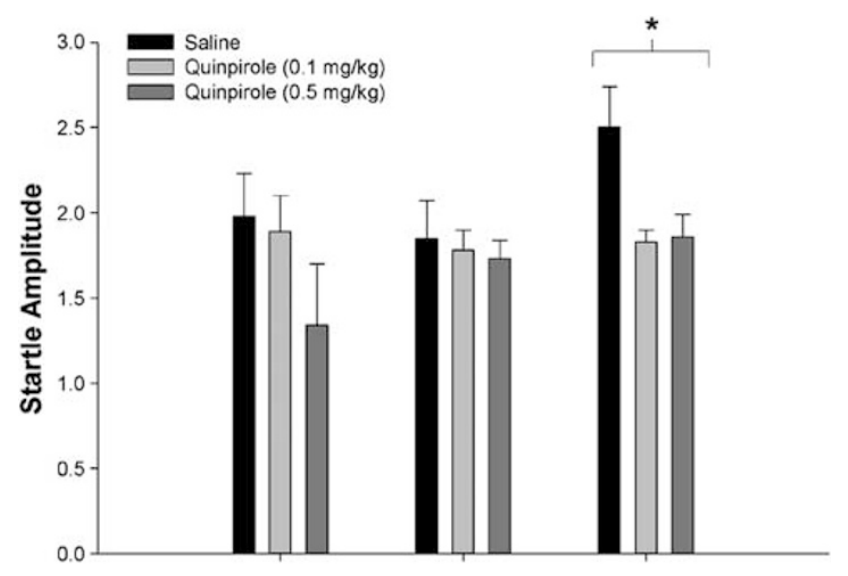

Figure 3 Mean startle amplitude $( \pm S E M)$ following saline or quinpirole treatment. $N=7-8$ /group. ${ }^{*} P<0.01$ as compared to DI and PPD2.

an attempt to determine whether the effects observed in PPD14 females were due to a shift in habituation processes. Thus, we examined changes in startle amplitude across the PPI testing procedure by examining the mean startle amplitude during blocks of pulse alone trials. These blocks consisted of three consecutive pulse alone trials that were interspersed throughout the 55 PPI stimulus trials. These blocks occurred on trials 12-14 (block 1), 30-32 (block 2) and 52-54 (block 3). A three-way repeated measure ANOVA was conducted with block as the within-subject factor and reproductive status and quinpirole dose as the between subject factors. These data are shown in Figure 4. There was an overall effect of block $(\mathrm{F}(2,420)=23.6, P<0.001)$ and a significant block $\times$ reproductive status $\times$ dose interaction $(\mathrm{F}(8,420)=2.35, \quad P<0.02)$, with no significant block $\times$ reproductive status or block $\times$ dose interactions. Post hoc analyses examining the three-way interaction indicated that this effect was due to several factors. First, the block effect (ie, decreased startle across blocks) was only significant when examined in subjects treated with quinpirole regardless of reproductive status (all $P$ 's $<0.01$ ). Second, the reproductive status effect was only significant when examined in saline-treated or low-dose quinpiroletreated females (all $P$ 's $<0.01$ ). Finally, while a significant drug effect was observed in PPD14 across all blocks, in DI females the only drug effect was only observed in block 3 and in PPD2 females the only drug effect was observed in block 2 (see Figure 4 for $P$-values).

Similar effects were observed in the between subject comparisons (ie, collapsed across blocks), with a significant main effect of reproductive status $(\mathrm{F}(2,210)=21.36$, $P<0.001)$ and dose $(\mathrm{F}(2,210)=17.65, P<0.001)$ as well as a reproductive status $\times$ dose interaction $(F(4,210)=6.06$, $P<0.001)$. Once again, the main effect of reproductive status was due to the increase startle amplitude observed in PPD14 females (all $P$ 's $<0.001$ ), while the main effect of dose was due to decreased startle amplitude following both doses of quinpirole (all $P$ 's $<0.001$ ). The significant interaction once again demonstrated that differences between PPD14 females and the other two reproductive status groups were due to increased startle amplitude in saline-treated PPD14 females, which was attenuated by treatment with the higher dose of quinpirole (all $P^{\prime} s<0.01$ ). These data indicate that 


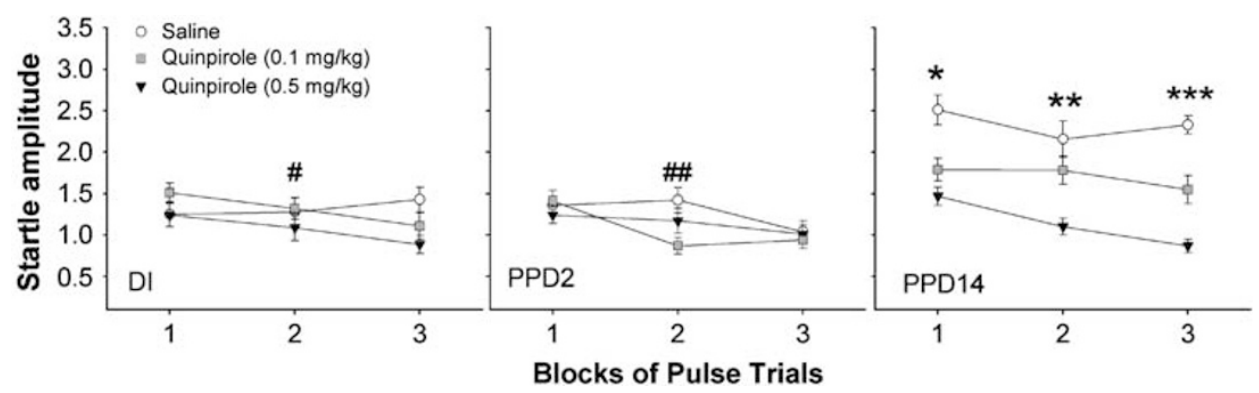

Figure 4 Mean startle amplitude $( \pm S E M)$ during blocks of pulse trials following either saline or quinpirole in each reproductive status group. $* P<0.00$ I saline vs 0.1 and $0.5 \mathrm{mg} / \mathrm{kg}$ quinpirole; ${ }^{*} * P<0.01$, saline and $0.1 \mathrm{mg} / \mathrm{kg}$ vs $0.5 \mathrm{mg} / \mathrm{kg}$ quinpirole; ${ }^{*} * * *<0.01$, all groups differ from one another; ${ }^{\#} P<0.05$, saline vs $0.5 \mathrm{mg} / \mathrm{kg}$ quinpirole; ${ }^{\#} \mathrm{P}<0.05$, saline vs $0.1 \mathrm{mg} / \mathrm{kg}$ quinpirole.

mid-lactation, but not early lactation, is associated with an enhanced startle response, an effect observed throughout the entire testing procedure and which is attenuated by administration of the D2 agonist quinpirole.

\section{Prepulse Inhibition}

The effects of reproductive status and quinpirole dose on PPI are illustrated in Figure 5. Once again a two-way ANOVA was conducted with reproductive status and quinpirole dose as factors. A significant main effect of reproductive status was observed $(\mathrm{F}(2,67)=4.08, P<0.025)$ as well as a trend toward a main effect of quinpirole dose $(\mathrm{F}(2,67)=2.64, P=0.08)$ with no interaction $(P=0.31)$. The effect of reproductive status was due to significantly decreased levels of PPI in PPD2 as compared to DI controls. Upon examination of the data, however, it appeared that the reduced basal PPI observed in the postpartum females (ie, saline-treated PPD2 and PPD14 females) may have confounded the comparisons between the reproductive status groups following quinpirole treatment. Indeed, a one-way ANOVA on PPI in saline-treated subjects revealed a significant effect of reproductive status, with both postpartum groups having significantly reduced PPI when compared to DI controls (both $P$ 's $<0.05$ ). Therefore, additional one-way analyses were conducted to examine the simple main effects of quinpirole in each reproductive status group. These analyses indicated that while quinpirole significantly disrupted PPI in DI females, neither dose of quinpirole was capable of disrupting PPI in the lactating females.

\section{Dopamine and DOPAC Content and Brain Turnover}

Mean dopamine and DOPAC content in the nucleus accumbens and dorsal striatum following saline and quinpirole treatment are represented in Table 2. For each brain region, dopamine and DOPAC content (ng/mg protein) were subjected to two-way ANOVA with reproductive status and quinpirole dose as factors. Significant changes in DOPAC content were observed in the nucleus accumbens where there was a significant main effect of reproductive status $(\mathrm{F}(2,67)=3.65, P<0.032)$. Post hoc analyses revealed that DOPAC was significantly reduced in PPD2 females when compared to PPD14 females $(P<0.05)$. There was no main effect of quinpirole dose $(P=0.14)$ and no interaction $(P=0.32)$. A significant main effect of

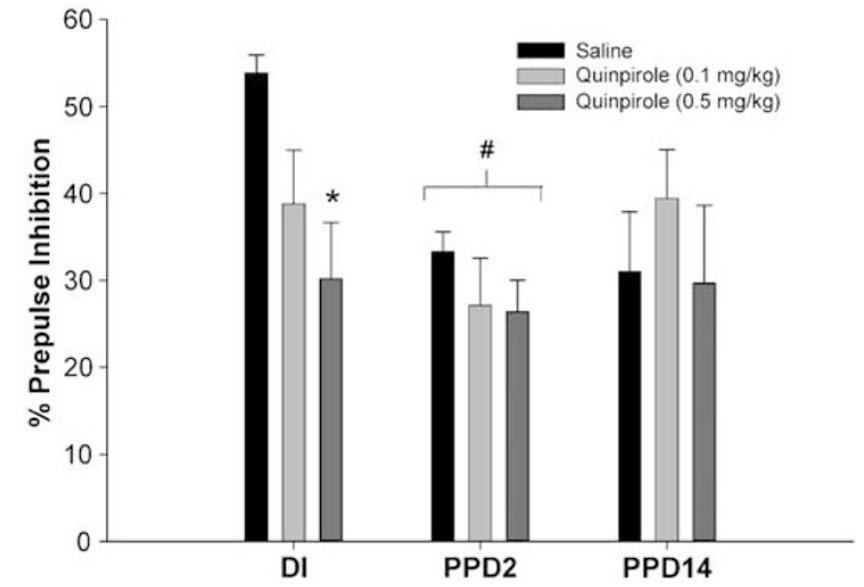

Figure 5 Mean PPI $( \pm S E M)$ following quinpirole treatment by reproductive status. $N=7$-8/group. ${ }^{\#} P<0.05$ as compared to DI controls. $* P<0.05$ as compared to saline within reproductive status.

quinpirole dose was observed for dopamine content within the nucleus accumbens $(\mathrm{F}(2,67)=7.81, P<0.001)$. Post hoc analyses indicated that increased dopamine content was observed following the higher dose of quinpirole $(0.5 \mathrm{mg} /$ $\mathrm{kg}$ ) when compared to either saline $(P<0.001)$ or the lower dose of quinpirole $(0.1 \mathrm{mg} / \mathrm{kg} ; P<0.02)$. In the dorsal striatum significant main effects on DOPAC content were observed for both reproductive status $(\mathrm{F}(2,66)=3.64$, $P<0.05)$ and quinpirole dose $(\mathrm{F}(2,66)=4.32, P<0.025)$ with no significant interaction $(P=0.72)$. Post hoc analyses indicated that the reproductive status effect was due to increased DOPAC content in PPD2 females as compared to PPD14 females $(P<0.05)$ while the quinpirole dose effect was due to decreased DOPAC content following the lower dose of quinpirole $(0.1 \mathrm{mg} / \mathrm{kg})$ as compared to both saline and the higher dose of quinpirole $(0.5 \mathrm{mg} / \mathrm{kg}$; both $P$ 's $<0.05)$. A significant main effect of reproductive status on dopamine content was observed the dorsal striatum, with no main effect of quinpirole dose $(P=0.24)$ and no interaction $(P=0.69)$. Post hoc analyses indicated that this reproductive status effect was due to increased dopamine content in PPD2 females when compared to both PPD14 and DI females (both P's $<0.01$ ). Thus, overall PPD2 females appear to have reduced DOPAC content in the nucleus accumbens, with increased DOPAC and dopamine content in the striatum. 
Table 2 Mean DOPAC and DA ng/mg Protein ( \pm SEM) from Nucleus Accumbens and Dorsal Striatum

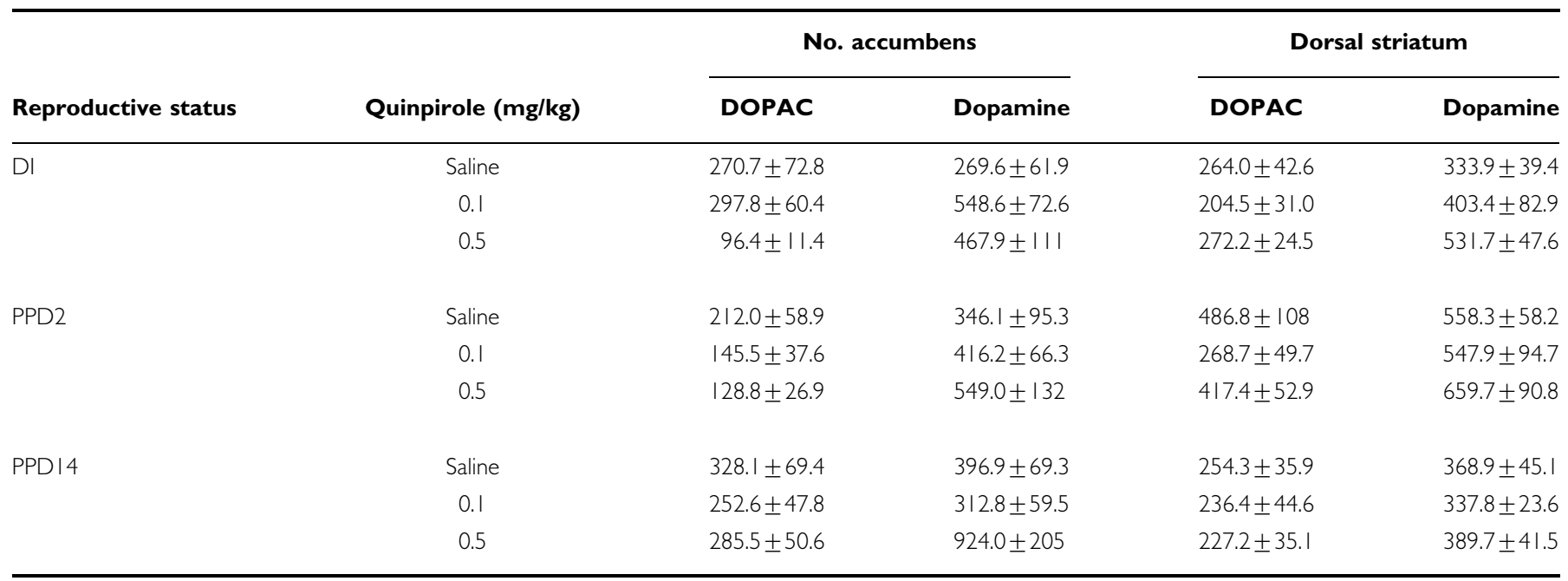

$N=7-8$ per group.

To determine the extent of dopamine turnover in the nucleus accumbens and dorsal striatum, we used the ex vivo DOPAC/dopamine ratio as a measure of dopamine utilization. A two-way ANOVA was used to determine the effects of reproductive status and quinpirole dose on dopamine utilization in the nucleus accumbens. The analysis revealed significant main effects of reproductive status $(\mathrm{F}(2,67)=6.75, \quad P<0.002)$ and quinpirole dose $(\mathrm{F}(2,67)=22.5, P<0.001)$ as well as a significant interaction $(\mathrm{F}(4,67)=3.37, P<0.02)$. As shown in Figure 6 (top panel) the main effect of reproductive status was due to a reduction in turnover observed in PPD2 females when compared to both PPD14 $(P<0.01)$ and DI $(P<0.02)$ females. The main effect of quinpirole dose was due to a significant dose dependent reduction in dopamine turnover following quinpirole (all $P$ 's $<0.01$ ). Finally, the significant interaction was based on the differential dose-response effects observed in the three reproductive groups with both postpartum groups being less sensitive to the lower dose of quinpirole.

The initial two-way ANOVA performed on dorsal striatal dopamine turnover was not normally distributed, therefore data were transformed using the following formula (1/-DOPAC/dopamine ratio) after which all data passed normality. A two-way ANOVA was then conducted on the transformed data, however, the data presented in Figure 6 (bottom panel), are the non-transformed data to allow for comparison with findings from the nucleus accumbens. The analysis revealed a significant main effect of quinpirole dose $(\mathrm{F}(2,67)=3.75, P<0.03)$ with no reproductive status effect $(P=0.34)$ and no interaction (0.52). The post hoc analyses indicated that the dose effect was due to decreased turnover following the lower dose of quinpirole $(0.1 \mathrm{mg} / \mathrm{kg})$ as compared to saline $(P<0.025)$.

\section{Brain cAMP Content}

Cyclic AMP was studied as a measure of intracellular change associated with each treatment condition. Figure 7 illustrates the effects of reproductive status and quinpirole
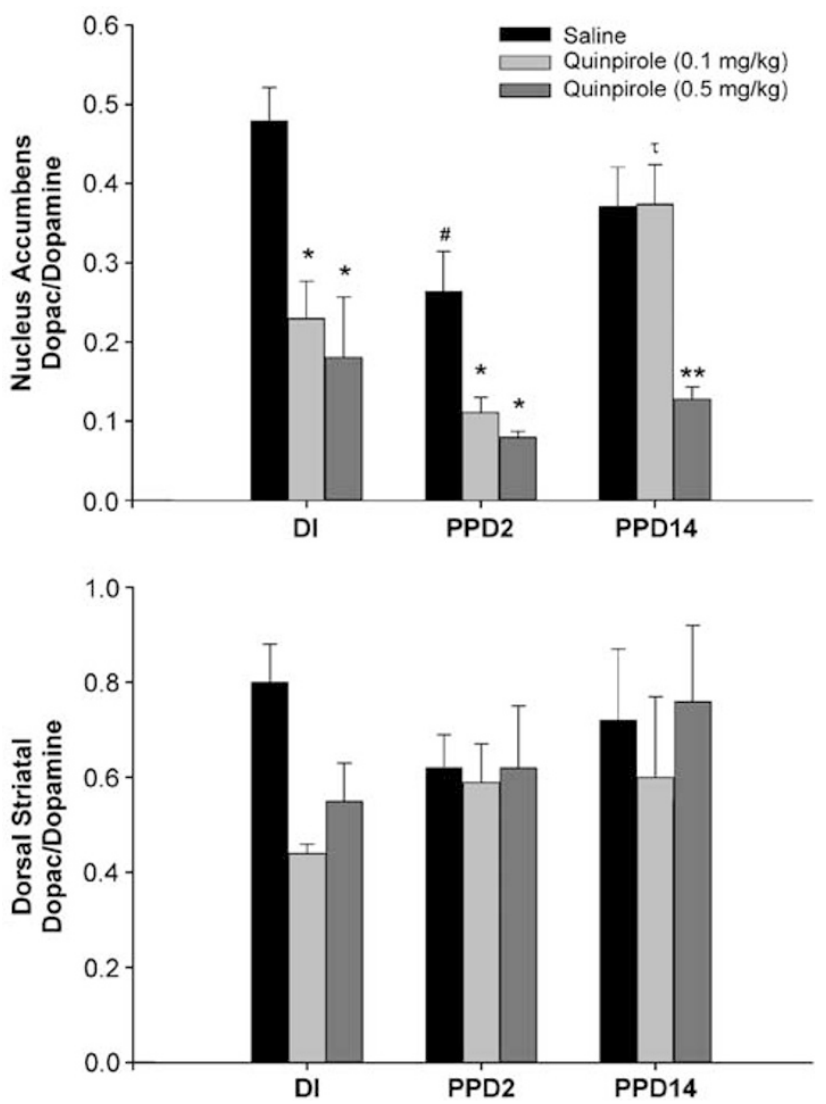

Figure 6 Upper panel: mean DOPAC/Dopamine ratio $( \pm$ SEM) within the nucleus accumbens following either saline or quinpirole treatment. Lower panel: mean DOPAC/dopamine ratio $( \pm \mathrm{SEM})$ in dorsal striatum following either saline or quinpirole treatment. $N=7-8$ /group. $* P<0.0$ I as compared to saline within reproductive condition; $* * P<0.01$ as compared to saline and $0.1 \mathrm{mg} / \mathrm{kg}$ dose within reproductive status; ${ }^{\#} P<0.05$ as compared to DI controls within dose; ${ }^{\tau} P<0.01$ as compared to PPD2 within dose.

treatment on cAMP content in the nucleus accumbens and dorsal striatum. cAMP content is expressed in relative units. Thus, relative cAMP content in the nucleus accumbens 

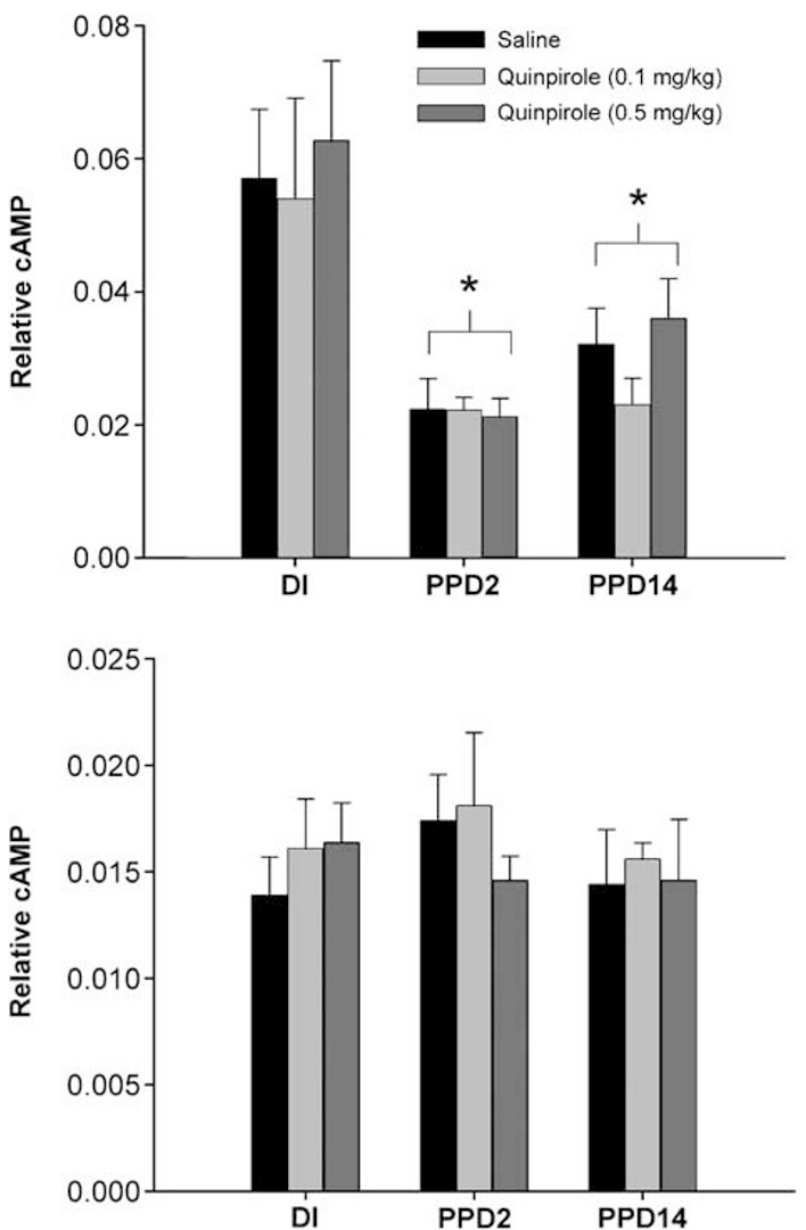

Figure 7 Upper panel: mean relative cAMP content $( \pm$ SEM) in the nucleus accumbens. Lower panel: mean relative cAMP content $( \pm$ SEM) in the dorsal striatum. $N=7-8 /$ group. $* P<0.05$ as compared to DI controls.

(upper panel) was subjected to two-way ANOVA with reproductive status and quinpirole dose as factors. The results indicate a main effect of reproductive status $(\mathrm{F}(2,73)=16.42, P<0.001)$ with significantly lower cAMP content in PPD2 and PPD14 animals compared to DI controls $(P$ 's $<0.001)$. There were no main effects of quinpirole dose, nor were any interactions between reproductive status and quinpirole dose revealed. For the dorsal striatum (lower panel), a two-way ANOVA revealed no effects of reproductive status, quinpirole dose, or their interaction. The data indicate that in the nucleus accumbens, basal (saline-treated) cAMP content is decreased during early and mid-lactation. This effect is not altered by acute quinpirole exposure and is not observed in the dorsal striatum.

\section{Plasma Corticosterone}

Plasma corticosterone levels were used as an additional measure of dopamine D2 receptor sensitivity as well as a physiological marker of stress in response to the testing paradigm. A two-way ANOVA with reproductive status and drug dose as factors was conducted on plasma corticosterone levels, as shown in Figure 8. This analysis revealed significant main effects of reproductive

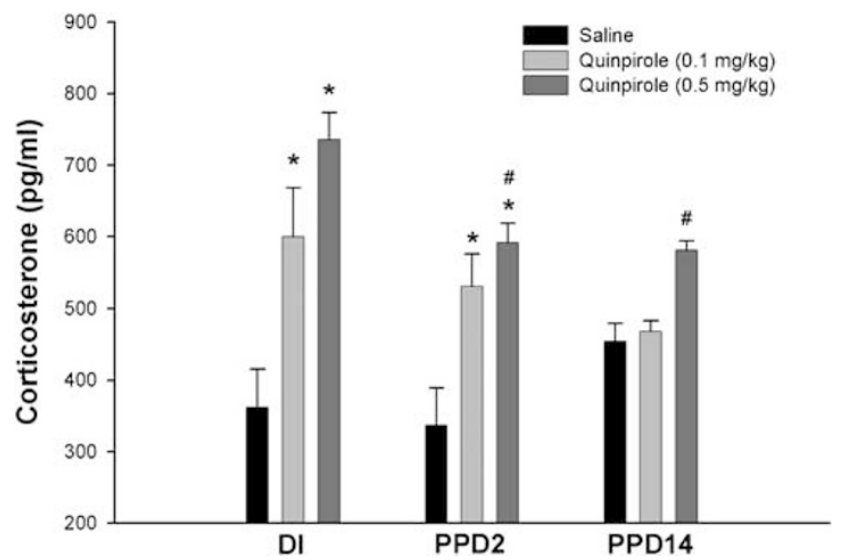

Figure 8 Mean $( \pm S E M)$ plasma corticosterone levels after the completion of behavioral testing in subjects treated with either saline or quinpirole. $N=7-8 /$ group. ${ }^{*} P<0.01$ as compared to saline-treated within reproductive status. ${ }^{\#} P<0.025$ as compared to Dl controls within dose.

status $(\mathrm{F}(2,71)=3.34, \quad P<0.05)$ and quinpirole dose $(\mathrm{F}(2,71)=27.14, P<0.001)$ as well as a significant reproductive status $\times$ dose interaction $(\mathrm{F}(4,71)=3.59, P<0.02)$. Post hoc analyses indicated that in the DI group, both doses of quinpirole significantly increased plasma corticosterone (Both $P^{\prime}$ s $<0.001$ as compared to saline). Quinpirole also increased plasma corticosterone in the PPD2 group, although this effect only reached statistical significance at the higher dose $(P<0.001)$. Neither dose of quinpirole significantly increased corticosterone in the PPD14 group (both $P$ 's $=0.11$ as compared to saline). Finally, a comparison of plasma corticosterone levels across reproductive status group revealed a significant difference between DI and PPD14 groups at the lower dose of quinpirole $(P<0.05)$, as well as differences between DI and both PPD2 and PPD14 at the higher dose of quinpirole (both $P$ 's $<0.05)$. These data confirm the effects of dopamine D2 receptor stimulation on corticosterone secretion in cycling rats and indicate decreased sensitivity to the effect of quinpirole on this response during lactation.

\section{Plasma Estradiol and Progesterone}

To confirm changes in ovarian hormone secretion during lactation, plasma estradiol and progesterone were measured. A two-way ANOVA was used to determine the effects of reproductive status and quinpirole dose. A significant effect of reproductive status on plasma estradiol levels was revealed $(\mathrm{F}(2,64)=17.09, P<0.001)$ which was due to significant decreases in PPD2 and PPD14 as compared to DI controls ( $P$ 's $<0.001$; Figure 9). There was no effect of quinpirole treatment and no interaction.

Analysis of progesterone levels also revealed a significant main effect of reproductive group in saline-treated subjects $(\mathrm{F}(2,69)=112.37, P<0.001)$ which was due to higher levels observed in the PPD14 group as compared to both the PPD2 group and DI controls ( $P$ 's $<0.001$; Figure 9). While there was no main effect of quinpirole dose on plasma progesterone $(P=0.3)$, there was a significant reproductive status $\times$ dose interaction $(F(4,69)=3.26, P<0.02)$. This interaction was due to the dose-dependent increase in plasma progesterone observed in DI controls. No effect of 

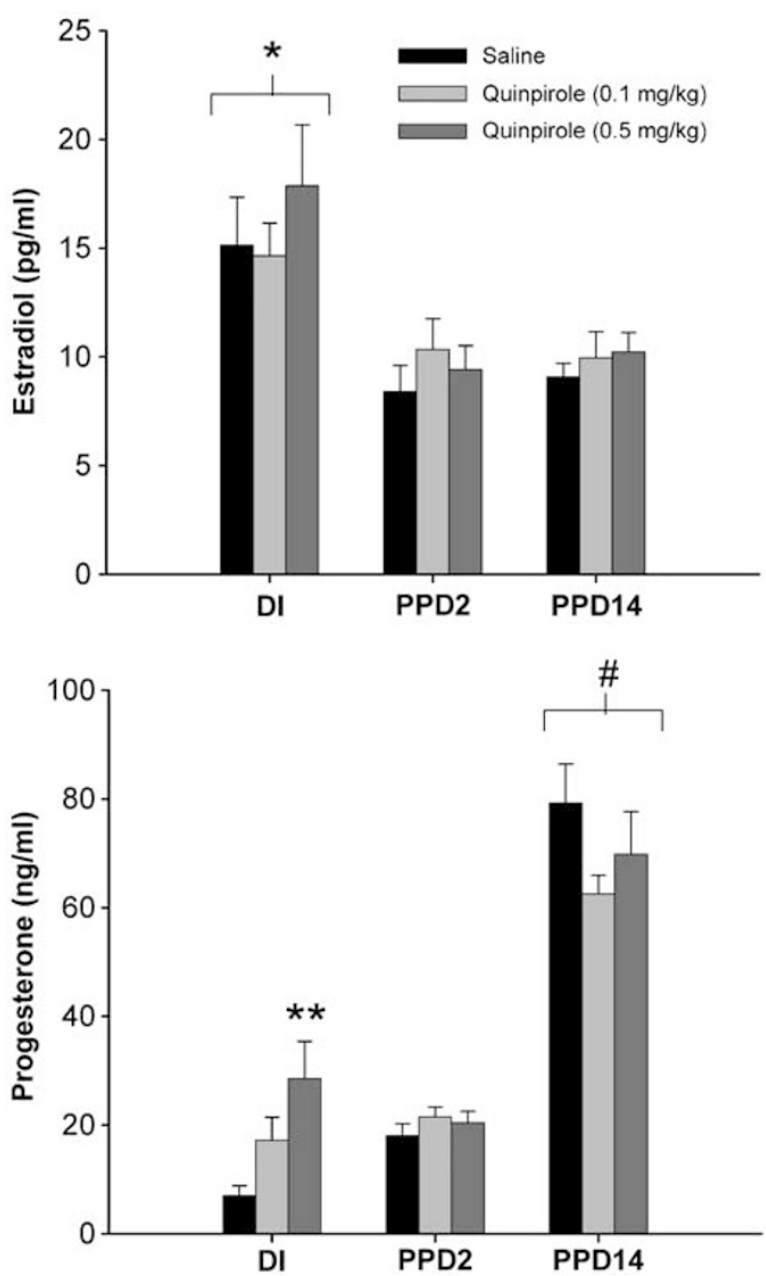

Figure 9 Upper panel: mean estradiol levels ( \pm SEM) following either saline or quinpirole treatment by reproductive status. $N=7-8 /$ group. ${ }^{*} P<0.001$ as compared to PPD2 and PPDI4. Lower panel: mean progesterone levels $( \pm$ SEM) following either saline or quinpirole treatment by reproductive status. $N=7-8$ /group. ${ }^{\#} P<0.00$ I as compared to DI and PPD2. ${ }^{*} P<0.05$ as compared to saline-treated group within DI.

quinpirole, however, was observed in either of the lactating groups. Thus, while higher levels of progesterone were only observed in the PPD14 groups, neither lactating group was responsive to quinpirole-mediated increases in plasma progesterone.

\section{DISCUSSION}

The current study observed significant alterations in both sensorimotor gating and dopamine function during the postpartum period. These changes included shifts in both the acoustic startle response as well as PPI. Alterations in DOPAC and dopamine content, cAMP production and D2-receptor sensitivity were also observed in postpartum females. These changes were not always uniform, with differing effects observed as a function of both the neuroanatomical region and postpartum time point examined.

In both of the postpartum time points we studied, a significant reduction in PPI was observed. This effect was not associated with increased sensitivity of the dopamine D2-receptor, or with increased dopamine turnover within the nucleus accumbens. Indeed, PPD2 females had reduced dopamine turnover in the nucleus accumbens and both postpartum groups demonstrated decreased D2-receptor sensitivity on several parameters. A previous study examining PPI and dopamine receptor sensitivity found that several weeks after weaning female rats with prior reproductive experience had normal basal PPI but demonstrated increased sensitivity to the disruptive effects of the mixed dopamine agonist apomorphine (Byrnes et al, 2001). Together with the current findings, these earlier results suggest that changes in neural dopamine function observed during the postpartum period may lead to long-term modifications in postsynaptic receptor sensitivity in parous females. It would be interesting to determine whether similar alterations in PPI and dopamine function are observed in a subsequent pregnancy or whether these effects are confined to a female's first postpartum experience.

Several studies have demonstrated reduced PPI during phases of the menstrual/estrous cycle during which either estrogen or estrogen and progesterone levels are elevated (ie, late follicular and mid-luteal in women, proestrus in rats) (Jovanovic et al, 2004; Koch, 1998; Swerdlow et al, 1997). Other studies, however, have demonstrated enhanced PPI following estradiol administration (Van den Buuse and Eikelis, 2001). In the present study, both groups of postpartum females had reduced levels of circulating estradiol but differed significantly with regard to levels of progesterone and yet both groups demonstrated reduced PPI. While it is possible that the rapid withdrawal and/or low levels of estrogen postpartum underlie the alterations in PPI, it is important to recognize that these data are correlative. At this time the precise role of circulating hormones on both the neural and behavioral changes observed postpartum remain to be determined.

The reduction in basal PPI observed postpartum also correlates with a decrease in basal levels of cAMP within the nucleus accumbens. The disruptive effects of quinpirole within the nucleus accumbens require functional $G_{i}$ and $G_{o}$ proteins, indicating that activation of inhibitory $\mathrm{G}$ proteins decreases PPI (Culm et al, 2003). Thus, it is possible that the reduction in cAMP accumulation observed in postpartum females is critical for the decreased levels of PPI observed postpartum. Several transmitter systems present within the nucleus accumbens are known to both regulate cAMP accumulation and have effects on PPI, these include but are not limited to glutamate, opioids, norepinephrine, as well as serotonin. The dissociation between reduced dopamine levels and disrupted PPI in postpartum females suggests that another transmitter system (or perhaps more than one), may be mediating this effect. Unfortunately studies examining changes in transmitter levels and/or second messenger function in the nucleus accumbens postpartum are lacking, therefore speculation as to which transmitter system may be involved in the decreased cAMP accumulation and how such changes may be related to PPI would be difficult. Future studies regarding the mechanisms underlying the reduction in sensorimotor gating in postpartum females and how they related to the reduction in cAMP levels are warranted. 
Alterations in acoustic startle amplitude were also observed in the current study, with PPD14 females displaying higher startle amplitudes following saline-treatment. This effect does not appear to be due to body weight differences as there was no correlation between body weight and startle amplitude and the lowest startle amplitudes were actually observed in the heaviest females. These data are in contrast to a previous study in lactating females which demonstrated reduced startle amplitudes in PPD14 females as compared to cycling females (Toufexis et al, 1999). Those authors suggested that the decreased startle amplitude may have been related to a reduction in anxiety or emotional responsiveness in lactating females. Indeed, acoustic startle responses are often used as a measure of anxiety-like behavior. We do not believe that the increased startle amplitude observed in PPD14 females observed in the current study is related to increased anxiety or emotional reactivity for several reasons. First, when PPD14 subjects were tested for their responses in an open field, no differences in the percent of time spent in the center of the open field were observed. Second, no significant differences in corticosterone levels were observed in saline-treated females, suggesting that the stress of the testing procedure was similar in all three reproductive status groups. Finally, PPD14 females treated with quinpirole demonstrated startle amplitudes similar to those observed in the other reproductive status groups. As quinpirole has no known anxiolytic effects and had no effect on the percent of time spent in the center of the open field, this would argue against the hypothesis that differences in emotional responsiveness underlie the observed changes in startle amplitude. The enhanced acoustic startle response, however, was observed throughout the entire testing procedure. This suggests that PPD14 females fail to habituate to the acoustic stimulus. Treatment with quinpirole reversed this effect. Thus, it is possible that a reduction in sensitivity of the dopamine $\mathrm{D} 2$ receptor may underlie the enhanced startle response observed during mid-lactation.

Postpartum changes in the sensitivity to quinpirole were observed on several measures in addition to PPI. Postpartum females demonstrated reduced sensitivity to quinpirole's effects on dopamine turnover within the nucleus accumbens, with the low dose of quinpirole having no effect on the dopamine turnover in these females, while significantly reducing turnover in diestrus controls. Reduced D2-receptor sensitivity was also observed with regard to corticosterone secretion. Both systemic and intraventricular administration of quinpirole stimulates plasma corticosterone secretion (Borowsky and Kuhn, 1992; Foreman et al, 1989; Fuller et al, 1983). More recent work has demonstrated that the stimulatory effects of dopamine agonists on corticosterone secretion are mediated by activation within the ventral striatum (Ikemoto and Goeders, 1998). Both doses of quinpirole significantly increased plasma corticosterone in diestrous controls, while only the higher dose was effective in PPD2 females. In PPD14 females neither dose significantly increased corticosterone. While not statistically significant, however, the higher dose of quinpirole did appear to have some effect on plasma corticosterone in the PPD14 females, although the response was significantly lower than that observed in diestrous females. Finally, while a significant dose-dependent effect of quinpirole on circulating progesterone secretion was observed in diestrus females, no such effects were observed in postpartum females. Previous work in cycling females found that administration of adrenocorticotropin hormone (ACTH) stimulates the secretion of both corticosterone and circulating progesterone levels (Putnam et al, 1991). Thus, the effects of quinpirole on progesterone may be mediated via the stimulation of ACTH. In the PPD14 females the lack of a progesterone response following quinpirole could be due to a ceiling effect, given the high levels of progesterone. In PPD2 females, however, progesterone levels were not significantly different from diestrous controls, and the higher dose of quinpirole did stimulate corticosterone secretion, yet no effect of quinpirole on progesterone secretion was observed. Thus, a shift in the ability of quinpirole to alter progesterone secretion occurs postpartum which may be related to a general change in hypothalamic regulation of gonadotropin secretion in lactating females.

The role of dopaminergic dysfunction in the etiology of psychosis has been widely studied and it has been postulated to play a role in postpartum psychosis as well. The present study, using both behavioral and physiological measures, reveals a reduction in D2-receptor sensitivity in the postpartum female, which coincides with changes in forebrain dopamine content and second messenger function within the nucleus accumbens. These changes, however, are not uniform across the postpartum period with differing effects observed during the early $v s$ mid-postpartum period. It is possible that some of the differential effects may be related to the variation in circulating hormones. Estrogen levels are consistently low throughout the postpartum period, while progesterone is low on PPD2 but then significantly increases by PPD14. Recent data in males suggests a 'neuroleptic-like' effect of progesterone (Rupprecht et al, 1999). Thus, it is possible that the decreased D2-receptor sensitivity observed on PPD14 may be related the increased progesterone levels in these females.

It is interesting to note that the neural adaptation in response to pregnancy and parturition appears to be reduced PPI occurring in conjunction with decreased dopaminergic tone within the nucleus accumbens (eg, decreased turnover and reduced D2 receptor sensitivity). The reduction in sensorimotor gating observed postpartum may reflect a shift in neural processing of sensory inputs, perhaps suggestive of a hyper-reactive state that might be adaptive for a mother charged with protecting her young. Such enhanced reactivity may also subserve the increased startle response observed in PPD14. That these changes in sensorimotor gating and startle responses occur in the absence of enhanced dopaminergic function may be critical in preventing sensory overload and cognitive disorganization in the normal postpartum female. Postpartum psychosis may result from a failure in any one of these adaptive mechanisms.

\section{ACKNOWLEDGEMENTS}

This work was supported in part by Grants R01 HD39895 (RSB) and P30 NS047243 (Dr F Rob Jackson). 


\section{REFERENCES}

Ahokas A, Aito M (1999). Role of estradiol in puerperal psychosis. Psychopharmacology 147: 108-110.

Ahokas A, Aito M, Rimon R (2000). Positive treatment effect of estradiol in postpartum psychosis: a pilot study. Journal of Clinical Psychiatry 61: 166-169.

Borowsky B, Kuhn CM (1992). D1 and D2 dopamine receptors stimulate hypothalao-pituitary-adrenal activity in rats. Neuropharmacology 31: 671-678.

Braff DL, Geyer MA, Swerdlow NR (2001). Human studies of prepulse inhibition of startle: normal subjects, patient groups, and pharmacological studies. Psychopharmacology 156: 234-258.

Byrnes EM, Byrnes JJ, Bridges RS (2001). Increased sensitivity of dopamine systems following reproductive experience in rats. Pharmacol Biochem Behav 68: 481-489.

Culm KE, Lim AM, Onton JA, Hammer RP (2003). Reduced Gi and Go protein function in the rat nucleus accumbens attenuates sensorimotor gating deficits. Brain Res 982: 12-18.

Foreman MM, Fuller RW, Hynes MD, Gidda JS, Nichols CL, Schaus JM et al (1989). Preclinical studies on quinelorane, a potent and highly selective D2-dopaminergic agonist. J Pharmacol Exp Therapeut 250: 227-235.

Fuller RW, Snoddy HD, Mason NR, Clemens JA, Bemis KG (1983). Elevation of serum corticosterone in rats by dopamine agonists related in structure to pergolide. Neuroendocrinology 36: $285-290$

Huber TJ, Rollnik J, Wilhelms J, von zur Muhlen A, Emrich HM, Schneider U (2001). Estradiol levels in psychotic disorders. Psychoneuroendocrinol 26: 27-35.

Ikemoto S, Goeders NE (1998). Microinjections of dopamine agonists and cocaine elevate plasma corticosterone: dissociation effects among the ventral and dorsal striatum and medial prefrontal cortex. Brain Res 814: 171-178.

Jovanovic T, Szilagyi S, Chakravorty S, Fiallos AM, Lewison BJ, Parwani A et al (2004). Menstrual cycle phase effects on prepulse inhibition of acoustic startle. Psychophysiology 41: 401-406.

Kaplan GB, Sethi RK, McClelland EG, Leite KA (1998). Regulation of $G$ protein-mediated adenylyl cyclase in striatum and cortex of opiate-dependent and opiate withdrawing mice. Brain Res 788: $104-110$

Koch M (1998). Sensorimotor gating changes across the estrous cycle in female rats. Physiol Behav 64: 625-628.

Kohl C (2004). Postpartum psychoses: closer to schizophrenia or the affective spectrum? Curr Opin Psychiatry 17: 87-90.

Lightman SL, Young WS (1989). Lactation inhibits stress-mediated secretion of corticosterone and oxytocin and hypothalamic accumulation of corticotrophin-releasing factor and enkephalin messenger ribonucleic acids. Endocrinology 124: 2358-2364.

Meakin CJ, Brockinton IF, Lynch SE, Jones SR (1995). Dopamine supersensitivity and hormonal status in puerperal psychosis. Br J Psychiatry 166: 73-79.

Munirathiam G, Yoburn BC (1994). A simple procedure for assaying cAMP. Pharmacol Biochem Behav 48: 813-816.

Paxinos G, Watson C (1998). The Rat Brain in Stereotaxic Coordinates. Academic Press: New York.

Putnam CD, Brann DW, Mahesh VB (1991). Acute activation of the adrenocorticotropin-adrenal axis: effect on gonadotropin and prolactin secretion in the female rat. Endocrinology 128: $2558-2566$

Rupprecht R, Koch M, Montkowski A, Lancel M, Faulhaber J, Harting J et al (1999). Assessment of neuroleptic-like properties of progesterone. Psychophamacology 143: 29-38.

Seyfried LS, Marcus SM (2003). Postpartum mood disorders. Int Rev Psychiatry 15: 231-242.

Swerdlow NR, Braff DL, Masten VL, Geyer MA (1990). Schizophrenia-like sensorimotor gating abnormalities in rats following dopamine infusion into the nucleus accumbens. Psychopharmacology 101: 414-420.

Swerdlow NR, Hartman PL, Auerbach PP (1997). Changes in sensorimotor inhibition across the menstrual cycle: implications for neuropsychiatric disorders. Biol Psychiatry 41: 452-460.

Swerdlow NR, Martizez ZA, Hanlon FM, Platten A, Farid M, Auerbach $\mathrm{P}$ et al (2000). Toward understanding the biology of a complex phenotype: Rat strain and substrain differences in the sensorimotor gating-disruptive effects of dopamine agonists. J Neurosci 20: 4325-4336.

Toufexis DJ, Rochford J, Walker CD (1999). Lactation-induced reduction in rats' acoustic startle is associated with changes in noradrenergic neurotransmission. Behav Neurosci 113: $176-184$.

Van den Buuse M, Eikelis N (2001). Estrogen increases prepulse inhibition of acoustic startle in rats. Eur J Pharmacol 425: 33-41.

Wan FJ, Geyer MA, Swerdlow NR (1994). Accumbens D2 modulation of sensorimotor gating in rats: assessing anatomical localization. Pharmacol Biochem Behav 49: 115-163.

Wan FJ, Swerdlow NR (1993). Intra-accumbens infusion of quinpirole impairs sensorimotor gating of acoustic startle in rats. Psychopharmacology 113: 103-109.

Woodside B, Abizaid A, Caporale M (1998). The role of specific macronutrient availability in the effect of food restriction on length of lactational diestrus in rats. Physiol Behav 64: 409-414. 